\section{A3.18 SYNOVIAL FIBROBLASTS INHIBIT INFLAMMATORY T CELL RESPONSES THROUGH TRYPTOPHAN METABOLISM}

doi:10.1136/annrheumdis-2013-203216.18

'Lars-Oliver Tykocinski, 'Anna-Marie Lauffer, 'Antonia Bohnen, 'Stefan Krienke, 2 rris Oezen, ${ }^{2}$ Ulrike Litzenburger, ${ }^{2}$ Michael Platten, ${ }^{1}$ Hanns-Martin Lorenz. ${ }^{1}$ Department of Medicine V, Division of Rheumatology, University of Heidelberg, Heidelberg, Germany; '2Experimental Neuroimmunology, German Cancer Research Center (DKFZ), Heidelberg, Germany

Background and Objectives It has been more and more accepted that fibroblasts participate in a dynamic interplay with other cells. Fibroblasts recruit immune cells and are able to influence their differentiation, proliferation, activation and survival. The development of rheumatoid arthritis (RA) is linked to functional changes of synovial fibroblasts (SFb) and a local infiltration of inflammatory cells, including B- and T cells. So far, little is known about the interaction of SFb and $\mathrm{T}$ cells. Here, we analysed the influence of normal SFb on the activation, proliferation and cytokine production of $\mathrm{T}$ cells in vitro.

Materials and Methods SFb were isolated from synovectomy tissue of patients with osteoarthritis. CD4 $+\mathrm{T}$ cells were stimulated with PHA and interleukin (IL)-2 in the presence or absence of fibroblasts, in direct contact or separated by a transwell chamber; their proliferation was measured by ${ }^{3} \mathrm{H}$-thymidine incorporation or by PKH-26 staining. Cytokine secretion was quantified by ELISA. Surface marker expression was analysed by flow cytometry and mRNA levels of matrix metalloprotease (MMP) 1, MMP3 and Indoleamine 2,3-dioxygenase (IDO) were quantified by real-time PCR. IDO activity was measured by kynurenine levels detected by HPLC and blocked by 1-methyl-L-tryptophan.

Results SFb strongly reduced the proliferation of activated CD4+ $T$ cells and the secretion of pro-inflammatory cytokines like interferon (IFN)-gamma, tumour necrosis factor (TNF)-alpha or IL-17. The suppression was consistent even when the $\mathrm{T}$ cells were separated by a transwell membrane. Interestingly, to produce suppressive mediators the SFb first needed to be stimulated by soluble factors released by activated T cells. The dynamic interplay between $\mathrm{T}$ cells and SFb was also shown by the fact that the SFb support the activation of $T$ cells at early time points of coculture. The coculture induced the expression of MMP1 and MMP3, as well as IDO mRNA and the production of nitric oxide (NO) by the SFb. Blockade of IDO, but not of MMPs or iNOS, completely abrogated the suppression of $\mathrm{T}$ cells, indicating that the inhibitory effect of the fibroblasts is mediated by tryptophan metabolism.

Conclusions Secreted products of activated T cells induce IDO expression in $\mathrm{SFb}$ which in turn leads to decreased proliferation and cytokine production of the activated $\mathrm{T}$ cells. This intercellular mechanism may play an important role in preventing inappropriate $T$ cell activation and in the termination of immune reactions. The insights gained from this study may help to define the malfunctions of pathogenic SFb of RA patients.

\section{A3.19 THE miR-148a IS INDUCED BY TWIST1 AND TBET AND PROMOTES THE SURVIVAL OF EFFECTOR MEMORY T HELPER 1 LYMPHOCYTES BY REGULATING THE PROAPOPTOTIC GENE BIM}

doi:10.1136/annrheumdis-2013-203216.19

'Claudia Haftmann, 'Anna-Barbara Stittrich, 'Kristyna Hradlikova, 'Zhuo Fang, 'Markus Bardua, 'Kerstin Westendorf, 1,2Gitta Anne Heinz, ${ }^{3}$ Thomas Häupl, ${ }^{3} \mathrm{~J}$ oachim Sieper, 'Hyun-Dong Chang, ${ }^{4}$ Nikolaus Rajewsky, ${ }^{5}$ Hans-Martin Jäck, ${ }^{1, *}$ Andreas Radbruch, 1.* Mir-Farzin Mashreghi. 'Deutsches Rheuma-Forschungszentrum (DRFZ) Berlin, Berlin, Germany; IInstitute of Molecular Immunology, Helmholtz Zentrum München, Munich, Germany; ${ }^{3}$ Charité Universitätsmedizin Berlin, Campus Charité Mitte, Berlin, Germany; ${ }^{4}$ Max-Delbrueck-Center for Moleculare Medicine Berlin, Berlin, Germany; ${ }^{5}$ Division of Molecular Immunology, Friedrich-Alexander University Erlangen Nuernberg, Erlangen, Germany

*Equal contribution
Background Repeatedly activated effector memory (EM) T helper 1 (Th1) cells in the context of autoimmunity and chronic inflammation exhibit a varity of differences in their cellular programme compared to regular EM Th1 cells. They function independently of activation signals and therefore escape physiological and therapeutical regulation. The acquirement of these properties is probably mediated by reduced expression of the pro-apoptotic protein Bim. Recent results suggest that microRNA (miRNA) mediated regulation of $\mathrm{Bim}$ may play an important role for the persistence of EM Th1 cells in autoimmune disease. Therefore, we aimed to identify miRNAs with the ability to suppress the expression of Bim in EM Th1 cells.

Material and Methods Assuming that Th1 cells involved in autoimmune inflammation have a history of repeated restimulation by persistent (auto-) antigens, we use in vitro generated acutely (once) and chronically (four times) activated murine EM Th1 cells. By using high-throughput sequencing of miRNA expression libraries, we have identified miRNAs being differentially expressed between once and repeatedly reactivated Th1 cells. By performing gain or loss of function experiments we examined the functional impact of miR-148a in chronically activated EM Th1 cells.

Results We found that among Th subsets chronically activated EM Th1 cells uniquely express microRNA-148a. MiR-148a regulates expression of the proapoptotic gene Bim leading to a decreased $\mathrm{Bim} /$ $B c 12$ ratio. When inhibiting miR-148a using antagomirs in Th1 cells Bim expression increases, leading to enhanced apoptosis and reduced expansion of repeatedly reactivated EM Th1 cells. Knockdown of Bim expression by siRNA in miR-148a antagomir treated cells restored viability of the Th1 cells. This clearly proofs that miR-148a controls viability exclusively by regulating Bim expression. T cells isolated from the synovium of arthritic patients exhibit elevated miR-148a expression. Interestingly, Tbet (Th1 master transcription factor) and Twist1 (marker for chronically activated EM Th1 cells) induce expression of miR-148a.

Conclusions Taken together the data imply that Tbet and Twist1, besides controlling pathogenicity of Th1 cells, also regulate the longevity in chronic inflammation via the miR-148a-Bim-axis. MiR-148 plays an important role for the survival of EM Th1 cells in autoimmunity and chronic inflammation, thus, represents a highly potent molecular target for therapeutical treatment.

\section{A3.20 TNF REGULATES CD3 $\zeta$ EXPRESSION OF T LYMPHOCYTES VIA SRC-LIKE ADAPTOR PROTEIN-DEPENDENT PROTEASOMAL DEGRADATION}

doi:10.1136/annrheumdis-2013-203216.20

'Barbara Érsek, 'Viktor Molnár, ${ }^{2}$ Andrea Balogh, 2 János Matkó, ${ }^{3}$ Andrew P Cope, 'Edit I Buzás, 'András Falus, ${ }^{1,4}$ György Nagy. ${ }^{1}$ Department of Genetics, Cell, and Immunobiology, Faculty of Medicine, Semmelweis University, 1089 Budapest, Hungary; ${ }^{2}$ Department of Immunology, Eötvös Loránd University, 1117 Budapest, Hungary; ${ }^{3}$ Division of Immunology, Infection and Inflammatory Disease, Academic Department of Rheumatology, Center for Molecular and Cellular Biology of Inflammation, King's College School of Medicine, King's College London, London WC2R 2LS, UK; 'Department of Rheumatology, Semmelweis University, Medical School, 1023 Budapest, Hungary

Background and Objectives although $\zeta$-chain downregulation of human $\mathrm{T}$ lymhocytes is common at sites of chronic inflammation, the precise mechanism of $\zeta$-chain regulation is not known. Src-like adaptor protein (SLAP) is a regulator of $\mathrm{T}$ cell activation; earlier data have reported that SLAP regulates immunoreceptor signalling. We studied the mechanism of CD3 $\zeta$-chain downregulation.

Materials and Methods $\mathrm{CD} 3 \zeta$ and SLAP protein levels of $\mathrm{T}$ lymphocytes were measured by Western blot. Jurkat cells were transiently transfected with siRNAs to silence SLAP, knockdown efficiency of the siRNAs was measured by real-time RT-PCR and by Western blot. For confocal microscopy experiments cells were transfected with eGFP-SLAP cDNA vector or control eGFP vector. 
The colocalisation between $\mathrm{CD} 3 \zeta$ and SLAP were measured by laser confocal microscopy. CD3 $\zeta$ mRNA was measured by quantitative real-time RT-PCR, IL-2 level was measured by ELISA.

Results in vitro TNF treatment of human T cells selectively, dose dependently and reversibly downregulates CD3 $\zeta$-chain expression and inhibits activation-induced IL-2 expression $(p<0.01)$. Inhibition of the proteasome prevented the effect of TNF on CD3 $\zeta$-chain expression. The colocalization of SLAP with CD3 $\zeta$-chain and the SLAP expression were enhanced by TNF treatment $(p<0.01$ and $p<0.05$, respectively). SLAP silencing with small interfering RNA inhibited the TNF-induced $\zeta$-chain downregulation. SLAP levels of the CD4 T cells, isolated from patients with rheumatoid arthritis were higher than that of the healthy donors' $(p<0.05)$. In addition, in vitro TNF treatment did not alter the SLAP expression of the CD4 lymphocytes of anti-TNF therapytreated RA patients.

Conclusions our present data suggest that TNF regulates $T$ cell activation during inflammatory processes, by altering CD3 $\zeta$-chain expression via a SLAP-dependent mechanism. Thereafter SLAPdependent regulation of $\mathrm{CD} 3 \zeta$-chain may have an important role in the fine control of TCR signalling during chronic inflammation. SLAP may have a role in the pathomechanism of RA.

\section{A3.21 TNF $\alpha$ INFLUENCES RasGRP1 AND RasGRP3 EXPRESSION LEVELS IN PBMC, B AND T CELLS}

doi:10.1136/annrheumdis-2013-203216.21

${ }^{1} \mathrm{ML}$ Golinski, ${ }^{1} \mathrm{M}$ Hiron, ${ }^{1} \mathrm{C}$ Guillou, ${ }^{1} \mathrm{C}$ Derambure, ${ }^{3} \mathrm{O}$ Boyer, ${ }^{2} \mathrm{X}$ Le Loët, ${ }^{2} \mathrm{O}$ Vittecoq, ${ }^{2} \mathrm{~T}$ Lequerré. 'INSERM U905, Institute for Biomedical Research, University of Rouen, Rouen, France; 'Department of Rheumatology, Rouen University Hospital \& INSERM U905, Institute for Biomedical Research, University of Rouen, Rouen, France; ${ }^{3}$ Department of Immunology, Rouen University Hospital \& INSERM U905, Institute for Biomedical Research, University of Rouen, Rouen, France

Background Rheumatoid arthritis (RA) is the most common inflammatory arthritis. B and T lymphocytes play a central role in the pathophysiology of RA. RasGRP is a member of the CDC25 family of Ras guanyl nucleotide exchange factors. RasGRP1 is expressed in $\mathrm{T}$ and $\mathrm{B}$ cells whereas RasGRP3 is only expressed in $\mathrm{B}$ cells. In previous studies, we have shown that RasGRP3 expression level significantly decreased in Peripheral blood mononuclear cells (PBMC) from RA patients responders to adalimumab after 3 months, leading to the question of TNF $\alpha$ involvement in pathways including RasGRP1 and RasGRP3.

Objectives To study TNF $\alpha$ effects on RasGRP1 and RasGRP3 expression levels in vitro.

Methods We measured by qRT-PCR, RasGRP1 and RasGRP3 expression levels, i) in PBMC from 3 healthy controls (HC), ii) in negative selected $B$ and $T$ cells from $P B M C$ isolated from 3 buffy coat. In each condition, cells were cultured with or without BCR or TCR stimulation for 4 days and TNF $\alpha$ was added for 24 or 48 hours. Immunofluorescence staining was performed to cheque the cell purity and $\mathrm{B}$ and $\mathrm{T}$ cells stimulation by flow cytometry. To test the functionals effects of RasGRP1 and RasGRP3 overexpression in $\mathrm{T}$ and $\mathrm{B}$ cells respectively, IL-2 production was measured by ELISA in T-cells, and Elk-1 expression level was measured by qRT-PCR in $B$ cells before and after TNF $\alpha$ stimulation. In addition, TNF $\alpha$ effects on cell proliferation were evaluated by $\left[{ }^{3} \mathrm{H}\right]$ thymidine incorporation by the $\mathrm{B}$ and $\mathrm{T}$ cells.

Results In B cells, TNF $\alpha$ induced an increase of RasGRP1 $(\mathrm{p}<0.001)$ and RasGRP3 $(\mathrm{p}<0.001)$ expression levels in absence of BCR stimulation. In the same way, in T cells, TNF $\alpha$ induced an increase of RasGRP1 $(\mathrm{p}<0.001)$ and RasGRP3 $(\mathrm{p}<0.001)$ expression levels in absence of TCR stimulation. Furthermore, TNF $\alpha$ induced a significantly increase of IL-2 production $(p<0.05)$ in unstimulated T cells and of Elk-1 expression level $(p<0.01)$ in unstimulated B cells. However, TNF $\alpha$ have no effects on B and T cells proliferation.

Conclusions This study suggests the RasGRP1 and Ras GRP 3 regulation by $T N F \alpha$, independently of $B$ and $T$ cells stimulation. The increasing of RasGRP3 and RasGRP1 in B and T cells specifically via TNF $\alpha$ binding on its receptors could promote the activation and proliferation of $\mathrm{B}$ and $\mathrm{T}$ cells by an independent antigen pathway. This second pathway could explain the maintenance of $B$ and T cells activation.

\section{A3.22 UPREGULATED MICRORNA-182 EXPRESSION IS ASSOCIATED WITH ENHANCED CONVENTIONAL CD4+ T CELL PROLIFERATION IN SLE}

doi:10.1136/annrheumdis-2013-203216.22

1,2Tobias Alexander, ${ }^{2}$ Claudia Haftmann, '2Lars Templin, 1,2Jens Humrich, 'Gerd-Rüdiger

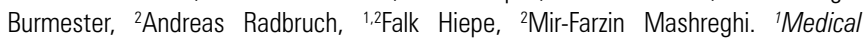
Department, Division of Rheumatology and Clinical Immunology, Charité - University Medicine Berlin, Germany; ${ }^{2}$ German Rheumatism Research Center (DRFZ), Berlin, Germany

Background Recent reports have shown dysregulated microRNAs (miRNAs) in murine models of lupus, among them increased expression of miRNA-182, which has been demonstrated to target the transcription factor FOXO1 in activated murine $\mathrm{CD}^{+} \mathrm{T}$ cells. The loss of FOXO1 activity in T cells is associated with spontaneous $\mathrm{T}$ cell activation, clonal expansion and autoantibody production, all of which are present in systemic lupus erythematosus (SLE).

Methods Expression levels of microRNA-182 (miR-182) and FOXO1 were analysed with RT-PCR in freshly isolated and magnetic purified peripheral blood $\mathrm{CD}^{+} \mathrm{T}$ cells from 9 patients with SLE and age/sex-matched healthy controls (HC). Multicolor flow cytometry was performed to analyse $\mathrm{CD}^{+} \mathrm{T}$ cell expression for CCR7, CD45RA, Ki-67, Foxp3, the interleukin-7 receptor- $\alpha$ (CD127) and phosphorylated STAT-5a (pSTAT5). Analysis of serum IL-7 levels was performed with ELISA in 27 SLE patients and HC (R\&D systems). The Wilcoxon signed-rank test was used for statistical analysis.

Results MiRNA-182 was significantly upregulated in $\mathrm{CD}^{+} \mathrm{T}$ cells from SLE patients compared to $\mathrm{HC}$ (median relative expression $8.89 \times 10 \mathrm{E}-7$ versus $3.96 \times 10 \mathrm{E}-7, p=0.008)$ while FOXO1 mRNA levels were decreased, yet without reaching statistical significance. Analysis of Ki-67 expression revealed an increased percentage of proliferating $\mathrm{CD}^{+}{ }^{+} \mathrm{T}$ cells in SLE $(5.23 \%$ versus $2.21 \%, p=0.006)$, which was more prominent in Foxp3 conventional T cells (Tcons) than in Foxp $^{+}$regulatory $\mathrm{T}$ cells (Tregs). Overall, $\mathrm{CD}^{+} \mathrm{T}$ cellular proliferation in SLE was associated with increased frequencies of CD45RA CCR7- effector memory T cells and enhanced basal pSTAT5 levels (median MFI 503.5 versus 399.0, $p=0.010$ ), suggesting a recent stimulation with common gamma chain $(\gamma c)$-signalling cytokines. In this regard, Tcons from SLE samples displayed decreased expression levels for the FOXO1 target gene CD127 (MFI 2021 versus 2553, $\mathrm{p}=0.049)$ and serum IL-7 levels were significantly higher in SLE when compared to HC (17.0 pg/ml versus $10.2 \mathrm{pg} / \mathrm{ml}, \mathrm{p}=0.001)$.

Conclusions MiR-182 expression has been shown to be directly dependent on STAT5 activation and to promote the clonal expansion of murine activated $\mathrm{CD}^{+} \mathrm{T}$ cells. Our data suggest that enhanced IL-7R/STAT5 signalling presumably mediates the induction of miR-182 expression, which in turn promotes the proliferation of Tcons in SLE. The relative contribution of IL-7R/miR-182/ FOXO1 axis on the enhanced proliferative capacity of SLE Tcons remains elusive and merit further investigation. Collectively, our data provide new insights in the pathophysiology of T cell hyperactivity in SLE and identifies miR-182 as a candidate target for future therapeutic approaches. 